\title{
Central Injection of Leptin Increases Sympathetic Nerve Outflows to the Stomach and Spleen in Anesthetized Rats
}

\author{
MAMORU TANIDA $^{1}$, YUSAKU IWASAKI $^{2}$ and NAOKI YAMAMOTO ${ }^{3}$ \\ ${ }^{1}$ Department of Physiology II, Kanazawa Medical University, Uchinada, Japan; \\ ${ }^{2}$ Laboratory of Animal Science, Graduate School of Life and Environmental Sciences, \\ Kyoto Prefectural University, Kyoto, Japan; \\ ${ }^{3}$ Department of Pharmaceutical Health Care, Faculty of Pharmaceutical Sciences, \\ Himeji Dokkyo University, Himeji, Japan
}

\begin{abstract}
Background/Aim: Leptin, one of the hormones produced in white adipose tissue, is an efferent sympathetic stimulator. Actually, an injection of leptin into the brain has been shown to activate sympathetic nerve activities innervating the kidney, adrenal gland, adipose tissues, liver, and lumbar in rats. Materials and Methods: This study investigated the effects of an intracerebroventricular injection of leptin on the activities of sympathetic nerves innervating the stomach and spleen in anesthetized rats. Results: Leptin injection activated the neural activities of sympathetic traffic to both the stomach and spleen. In addition, to investigate the role of AMP-activated protein kinase (AMPK), the effects of 5-aminoimidazole-4-carboxamide 1-beta-D-ribofuranoside (AICAR), an AMPK activator, or compound $C$, an AMPK inhibitor, on leptin-induced sympathoexcitation, were assessed. Central pretreatment with AICAR or compound C eliminated not only leptin-induced gastric sympathoexcitation but also leptin-induced splenic sympathoexcitation. Conclusion: Leptin stimulates efferent sympathetic outflow to the stomach and spleen through the hypothalamic AMPK.
\end{abstract}

Appetite is regulated by certain hormones, such as leptin, ghrelin, cholecystokinin, and peptide YY, released from peripheral organs (1). These hormones directly act on the hypothalamus or affect the brain by acting on afferent autonomic nerves in gastrointestinal organs (2). Leptin, a

This article is freely accessible online.

Correspondence to: Mamoru Tanida, Ph.D., Department of Physiology II Kanazawa Medical University, 1-1 Daigaku Uchinada Ishikawa 920-0293, Japan. Tel: +81 762188105, Fax: +81 762188010,e-mail: mtanida@kanazawa-med.ac.jp

Key Words: Efferent autonomic nerves, rats, hypothalamus, AMPK, gastrointestinal tract, spleen, leptin. hormone produced in white adipose tissue, acts on a certain area of the hypothalamus and regulates appetite, energy metabolism, cardiovascular function, and glucose metabolism (3). After the intake of food, increased leptin suppresses appetite by acting on the brain, resulting in sympathetic activation and alteration of gastric function. Central leptin has been reported to cause efferent sympathoexcitation of the kidney, adrenal grand, lumbar, liver, and brown and white adipose tissue in rats (4-7). With regard to gastric function, an intracerebroventricular (ICV) injection of leptin delayed gastric emptying in anesthetized rats $(8,9)$. In a recently published study, we demonstrated that the efferent sympathetic nerve supplying the stomach contributes to the modulation of gastric emptying through pyloric sphincter constriction in rats (10). However, whether central leptin affects the gastric sympathetic nerve activity (gastric SNA) has not yet been studied.

In contrast, leptin is also involved in the regulation of the immune system by activating lymphocytes (11). An ICV injection of leptin was found to suppress splenic lymphocyte functions via the sympathetic nerves in rats (12). However, the effect of central leptin on efferent splenic sympathetic nerve activity (splenic SNA) has not yet been examined.

In the present study, we first examined the effects of an ICV injection of leptin on gastric SNA and splenic SNA in anesthetized rats. The hypothalamic AMP-activated protein kinase (AMPK) acts not only as an energy sensor (13), but also as a regulator of efferent sympathetic nerves (14). Thus, we next examined the pharmacological role of AMPK in the gastric and splenic sympathetic responses to leptin injection.

\section{Materials and Methods}

Animals. Male Wistar rats weighing 244-276 g ( $\mathrm{n}=48)$ were housed in a room maintained at $23^{\circ} \mathrm{C}$ and illuminated for $12 \mathrm{~h}(08: 00-$ 20:00) every day. The rats were allowed access to food and water ad libitum. All animal care and handling procedures were approved by the Animal Research Committee of Kanazawa Medical University (nos. 2014-38). 
Intracerebroventricular cannulation. To examine the effect of an ICV injection of leptin or vehicle on the changes in sympathetic nerve discharge, a brain cannula composed of a metal pipe was inserted into the third cerebral ventricle using a stereotaxic apparatus (coordinates: AP, $1.0-\mathrm{mm}$ posterior to the bregma; $\mathrm{L}, 0$ $\mathrm{mm} ; \mathrm{V}, 7.5 \mathrm{~mm}$ ) and the atlas of Paxinos and Watson (15). The procedure was carried out under anesthesia by intraperitoneal injection of ketamine $(91 \mathrm{mg} / \mathrm{kg})$ and xylazine $(9.1 \mathrm{mg} / \mathrm{kg}), 7-10$ days before the Electrophysiological experiment.

Electro-physiological recording of efferent sympathetic nerve activity. The preparation procedures for the electrophysiological experiments were performed as described previously $(6,7,14)$. On the day of the experiment, the rats were deprived of food $3 \mathrm{~h}$ before surgery but were allowed free access to water. Under anesthesia, induced by intraperitoneal injection of $1.2 \mathrm{~g} / \mathrm{kg}$ urethane, following tracheal cannulation, polyethylene catheters were inserted in the left femoral vein of the rats for the administration of reagents. Another catheter was inserted in the left femoral artery for the measurement of blood pressure. To record gastric SNA and splenic SNA, a midline abdominal incision was made to expose the sympathetic branches on the gastric and splenic arteries, which were then identified using a microscope. Nerve bundles were cut on the proximal side to record efferent nerve activities. Each nerve bundle was attached to a pair of stainless-steel wire electrodes and fixed with silicone gel to prevent dehydration and provide electrical insulation. Electrical signals from nerves were amplified, filtered, monitored on an oscilloscope, and converted to standard pulses using a window discriminator. Both counted discharge rate and neural raw data were sampled using a Power-Lab analog-to-digital converter (8/30, AD Instruments, New South Wales, Australia) and stored on a hard disk for offline analysis.

Baseline measurements of gastric SNA and splenic SNA were recorded for $5 \mathrm{~min}$ just before an ICV injection of $10 \mu \mathrm{l}$ vehicle (artificial cerebrospinal fluid) or leptin $(10 \mu \mathrm{g} / 10 \mu \mathrm{l}$ vehicle). After the injection, sympathetic nerve activity was recorded for $180 \mathrm{~min}$. To examine the role of AMPK in neural activity due to leptin, AICAR $(1 \mu \mathrm{mol} / 10 \mu \mathrm{l} 20 \%$ dimethyl sulfoxide in phosphate buffered saline) or compound $\mathrm{C}(10 \mu \mathrm{g} / 10 \mu \mathrm{l} 20 \%$ dimethyl sulfoxide in phosphate buffered saline) was administered $15 \mathrm{~min}$ prior to the leptin injection. At the end of the experiment, hexamethonium chloride $(10 \mathrm{mg} / \mathrm{kg})$ was administered intravenously to ensure that post-ganglionic efferent sympathetic nerve activity was recorded. The rats were euthanized with an intravenous overdose injection of pentobarbital sodium $(100 \mathrm{mg} / \mathrm{kg})$ after the completion of all experimental procedures.

Data analysis. All data are expressed as the means \pm SEM. Two-way analysis of variance followed by Fisher's post-hoc test was used to compare differences in nerve activity between all groups. $p$-Values of $<0.05$ were regarded as statistically significant.

\section{Results}

The data recorded for gastric SNA and splenic SNA are shown in Figures 1A and 2A, respectively, before and after ICV injection of vehicle or leptin. Leptin injection increased gastric SNA and splenic SNA, whereas the injection of vehicle had no effect. As shown by the time-course data, 180 min post-injection (vehicle or leptin), leptin significantly elevated gastric SNA (Figure 1B) or splenic SNA (Figure 2B), $20 \mathrm{~min}$ or $10 \mathrm{~min}$ after injection, respectively. The highest levels of gastric SNA and splenic SNA after leptin injection were $166.6 \pm 19.5 \%$ and $189.0 \pm 24.2 \%$, respectively. To elucidate the role of hypothalamic AMPK, we examined the effects of an AMPK activator or AMPK inhibitor on the stimulatory responses of gastric and splenic SNA to leptin injection. Hypothalamic pre-injection of AICAR or compound $\mathrm{C}$ significantly eliminated gastric and splenic sympathoexcitation by leptin (Figures $1 \mathrm{C}$ and 2C). Additionally, the difference in basal values $(0 \mathrm{~min})$ between gastric SNA and splenic SNA was not statistically significant among all the groups (data not shown).

\section{Discussion}

In this study, we demonstrated, for the first time, that an ICV injection of leptin causes an increase in gastric SNA and splenic SNA in anesthetized rats. Additionally, leptin-induced gastric and splenic sympathoexcitation was abolished by pretreatment with the AMPK activator or AMPK inhibitor. Thus, to our knowledge, this study presents the first evidence that central leptin may stimulate efferent sympathetic outflow to the stomach and spleen through hypothalamic AMPK signaling.

Previous studies have shown that ICV injection of leptin delayed gastric emptying in anesthetized rats $(8,9)$. Moreover, this delay was blocked by an adrenergic ganglion blocker (8). Thus, it appears that efferent sympathetic traffic to the stomach may be involved in the leptin-induced delay in gastric emptying. This finding is further supported by the present study, in which gastric sympathoexcitation was observed after an ICV injection of leptin. The pyloric sphincter, modulated by intrinsic and extrinsic nerves of the autonomic nervous system, contributes to regulate the gastric emptying by changing the resistance of its own smooth muscle sphincter (16). In support of this idea, studies have shown that electrical stimulation of efferent sympathetic nerves innervating the pylorus cause contraction of the sphincter muscle $(16,17)$. We have recently observed that the stimulation of efferent gastric sympathetic nerves eliminated gastric contraction-induced gastric flux (10). This evidence indicates that increased gastric SNA inhibits gastric emptying. Thus, it is suggested that leptin-induced gastric sympathoexcitation may elicit a delay in gastric emptying via contraction of the pyloric sphincter.

In a previous study, central leptin suppressed the proliferative response of splenic lymphocytes to Concanavalin A via the sympathetic nervous system (12). However, the effect of central leptin on the efferent sympathetic discharge to the spleen has not yet been investigated. In this study, we showed for the first time, that an ICV injection of leptin 
A
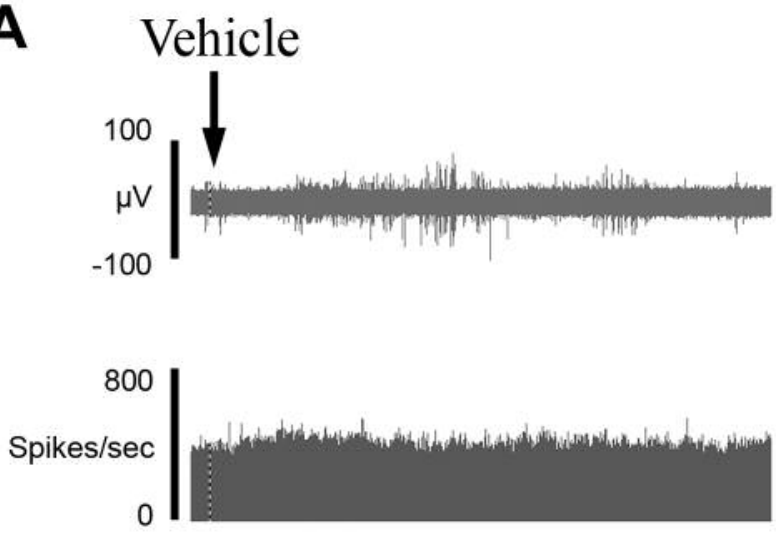

$90 \mathrm{~min}$

B

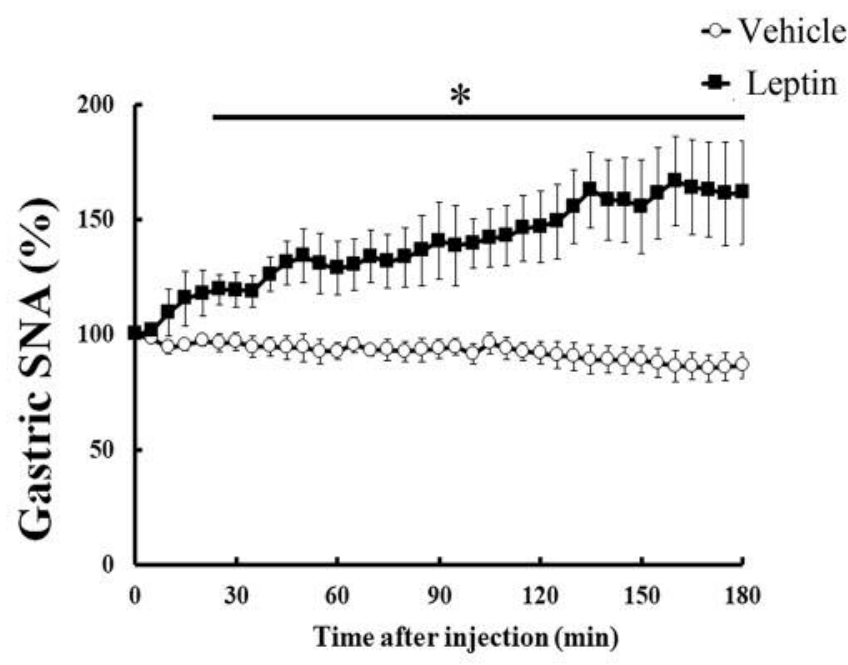

C

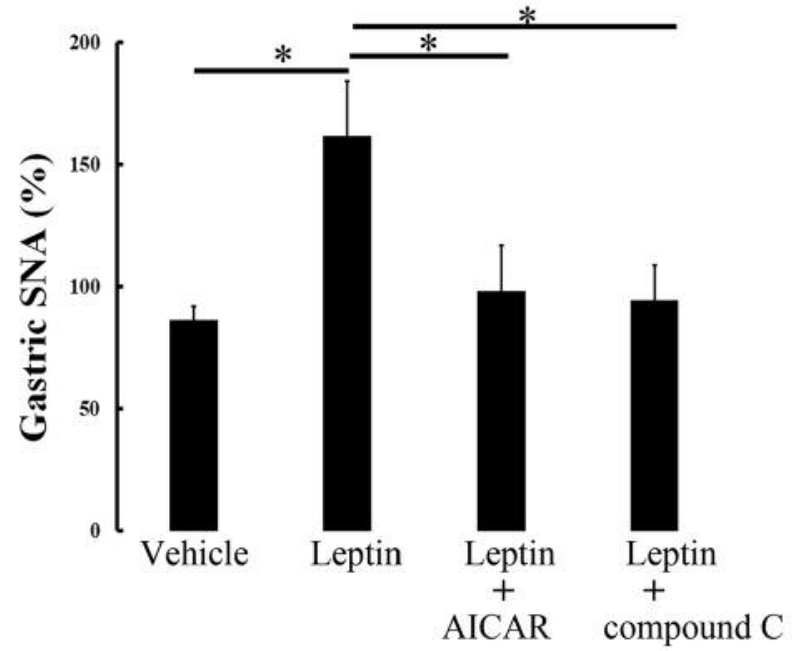

Figure 1. Effect of ICV injection of vehicle or leptin on gastric SNA. Representative recording data of gastric SNA before and after ICV injection of vehicle or leptin (A). Time-course change in gastric SNA after injection of vehicle $(n=7)$ or leptin $(n=6)$ is expressed as mean \pm SEM of percentages of values at $0 \mathrm{~min}(B) .{ }^{*} p<0.05$ vs. vehicle group. Bar graph shows responses of gastric SNA value 180 min after injection of vehicle and leptin (C). AICAR $(n=7)$ or compound $C(n=6)$ was injected before leptin injection. ${ }^{*} p<0.05$ vs. leptin group. 

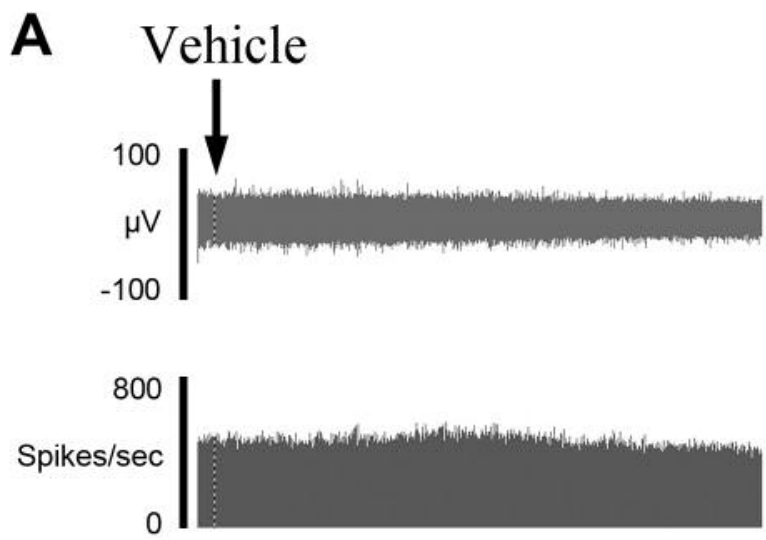
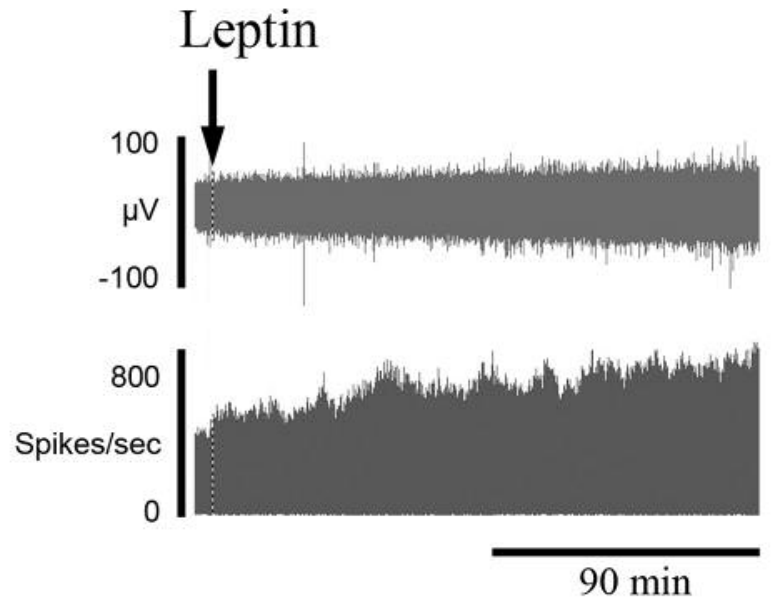

B

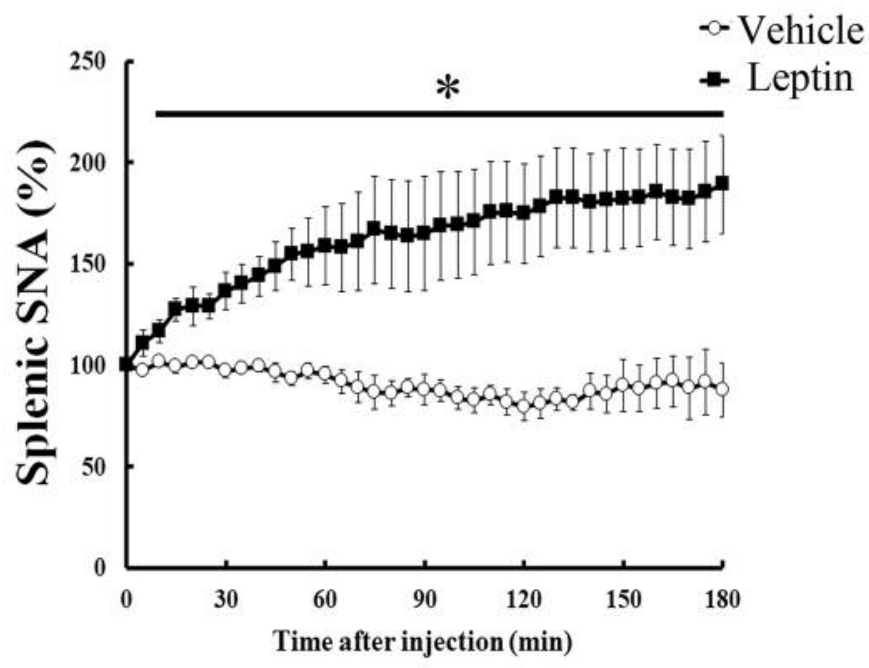

C

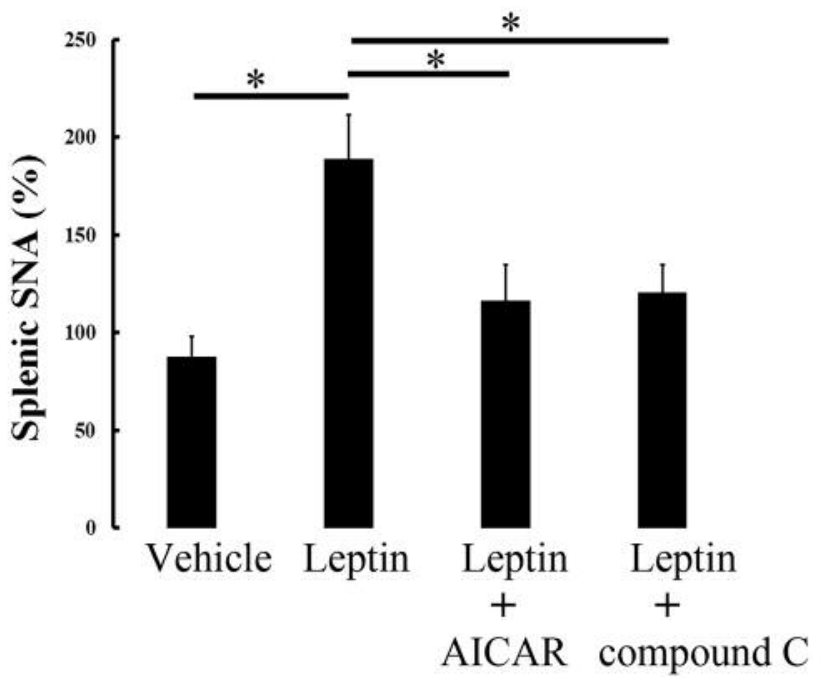

Figure 2. Effect of ICV injection of vehicle or leptin on splenic SNA. Representative recording data of splenic SNA before and after ICV injection of vehicle or leptin (A). Time-course change in splenic SNA after injection of vehicle ( $n=5)$ or leptin ( $n=6)$ is expressed as mean \pm SEM of percentages of values at $0 \mathrm{~min}(B) . * p<0.05 \mathrm{vs}$. vehicle group. Bar graph shows responses of splenic SNA value 180 min after injection of vehicle and leptin $(C)$. AICAR $(n=5)$ or compound $C(n=6)$ was injected before leptin injection. $* p<0.05$ vs. leptin group. 
increases splenic SNA in anesthetized rats, thus supporting the above idea. In contrast, in a rat experiment, increased sympathetic nerve activity reduced splenic natural killer cell activity (18). Thus, it appears that the central leptin-induced increase in splenic SNA may suppress the natural killer cell activity in the spleen. However, leptin can directly act on natural killer cells that express the leptin receptor. Thus, on the contrary, the direct action of leptin can activate of natural killer cell activity (19). Therefore, it can be speculated that there are two mechanisms of leptin action on the natural killer cell activity in the spleen, suggesting that leptin action through sympathetic nerves is different from the direct action of leptin. Thus, to clearly understand why the different effects of leptin on splenic natural killer cell activity were elicited via two pathways, additional experiments need to be conducted in the future.

The current study investigated the hypothalamic mechanism of central leptin actions on gastric and splenic SNA and demonstrated that pharmacological inhibition or activation of the hypothalamic AMPK eliminates the stimulatory responses of the gastric and splenic SNA to central leptin. Thus, hypothalamic AMPK may play an important role in leptin-induced sympathoexcitation in the stomach and spleen. Previously, we have shown that the hypothalamic AMPK also regulates leptin-induced renal sympathoexcitation and blood pressure elevation in rats $(6$, 14). The efferent sympathetic signal to the kidney is a baroreceptor reflex-related nerve that responds to sodium nitroprusside-induced hypotension (20). Gastric SNA (10) and splenic SNA (data not shown) also increased during hypotension after sodium nitroprusside injection. Thus, regarding efferent sympathetic regulation by central leptin, it is suggested that leptin may act on the hypothalamus and affect intracellular AMPK to regulate the efferent sympathetic activities projecting to the stomach, spleen and kidney, which participate in the baroreceptor reflex.

With respect to the eliminations of leptin-induced sympathoexcitations by pretreatment with AICAR or compound $\mathrm{C}$, there is no clear reason as to why both pharmacological activator and inhibitor attenuated leptin actions on efferent sympathetic traffics. However, a possible explanation includes that saturated sympathoexcitaion by pharmacological inhibition or activation of the AMPK activity may inhibit stimulatory responses of gastric and splenic sympathetic traffics to leptin injection since both ICV injection of ACIAR and compound $\mathrm{C}$ stimulated renal and adrenal sympathetic outflows in rats (14). Of course, in the present study, we could not examine the effects of AICAR and compound $\mathrm{C}$ on the gastric and splenic SNA, and further studies will be needed to certify the above hypothesis.

In conclusion, we found that an ICV injection of leptin stimulated gastric and splenic SNA in anesthetized rats. Hypothalamic AMPK may be implicated in the central leptin-induced sympathoexcitation of the stomach and spleen. These findings suggest that central leptin signals may contribute to the regulation of gastric function and the immune system through the neural pathway of hypothalamic AMPK and efferent sympathetic nerves. However, the detailed mechanism of how the central neural pathway from the hypothalamus to the medulla oblongata is affected during leptin-induced efferent sympathoexcitation has not been examined; therefore, further investigation is needed.

\section{Conflicts of Interest}

The Authors have declared that no competing interests exist regarding this study.

\section{Authors' Contributions}

M.T. conceived and designed research; M.T., Y.I., and N.Y. performed experiments, analyzed data, and interpreted the results of experiments; M.T. prepared figures and drafted the manuscript; M.T., Y.I., and N.Y. edited the manuscript and approved the final version of the manuscript.

\section{Acknowledgements}

This research was partially supported by grants (to M.T.) from the Ministry of Education, Culture, Sports, Science, and Technology of Japan (Grant-in-Aid for Young Scientists, 26870672), the Takeda Science Foundation, the Nestlé Nutrition Council Japan, and the Promoted Research of Kanazawa Medical University (S2014-2).

\section{References}

1 Larder R and O'Rahilly S: Shedding pounds after going under the knife: guts over glory-why diets fail. Nat Med 18(5): 666667, 2012. PMID: 22561823. DOI: 10.1038/nm.2747

2 Ueno $\mathrm{H}$ and Nakazato M: Mechanistic relationship between the vagal afferent pathway, central nervous system and peripheral organs in appetite regulation. J Diabetes Investig 7(6): 812-818, 2016. PMID: 27180615. DOI: 10.1111/jdi.12492

3 Francisco V, Pino J, Campos-Cabaleiro V, Ruiz-Fernández C, Mera A, Gonzalez-Gay MA, Gómez R and Gualillo O: Obesity, fat mass and immune system: Role for leptin. Front Physiol 9: 640, 2018. PMID: 29910742. DOI: 10.3389/fphys.2018.00640

4 Haynes WG, Morgan DA, Walsh SA, Mark AL and Sivitz WI: Receptor-mediated regional sympathetic nerve activation by leptin. J Clin Invest 100(2): 270-278, 1997. PMID: 9218503. DOI: $10.1172 / \mathrm{JCI} 119532$

5 Rahmouni K, Sigmund CD, Haynes WG and Mark AL: Hypothalamic ERK mediates the anorectic and thermogenic sympathetic effects of leptin. Diabetes 58(3): 536-542, 2009. PMID: 19066310. DOI: 10.2337/db08-0822

6 Tanida M, Yamamoto N, Shibamoto $\mathrm{T}$ and Rahmouni $\mathrm{K}$ : Involvement of hypothalamic AMP-activated protein kinase in leptin-induced sympathetic nerve activation. PLoS One $8(2)$ : e56660, 2013. PMID: 23418591. DOI: 10.1371/journal.pone. 0056660 
7 Tanida M, Yamamoto N, Morgan DA, Kurata Y, Shibamoto T and Rahmouni K: Leptin receptor signaling in the hypothalamus regulates hepatic autonomic nerve activity via phosphatidylinositol 3-kinase and AMP-activated protein kinase. J Neurosci 35(2): 474484, 2015. PMID: 25589743. DOI: 10.1523/JNEUROSCI.182814.2015

8 Cakir B, Kasimay O, Devseren E and Yeğen BC: Leptin inhibits gastric emptying in rats: role of CCK receptors and vagal afferent fibers. Physiol Res 56(3): 315-322, 2007. PMID: 16792473

9 Martínez V, Barrachina MD, Wang L and Taché Y: Intracerebroventricular leptin inhibits gastric emptying of a solid nutrient meal in rats. Neuroreport 10(15): 3217-3221, 1999. PMID: 10574563. DOI: 10.1097/00001756-199910190-00017

10 Kuda Y, Tanida M, Chen F, Kurata Y and Shibamoto T: Anaphylaxis stimulates afferent vagal nerve activity and efferent sympathetic nerve activity in the stomach of anesthetized rats. Am J Physiol Regul Integr Comp Physiol 317(2): R337-R345, 2019. PMID: 31116019. DOI: 10.1152/ajpregu.00193.2018

11 Lord GM, Matarese G, Howard JK, Baker RJ, Bloom SR and Lechler RI: Leptin modulates the T-cell immune response and reverses starvation-induced immunosuppression. Nature 394(6696): 897-901, 1998. PMID: 9732873. DOI: 10.1038/29795

12 Okamoto S, Irie Y, Ishikawa I, Kimura K and Masayuki Saito: Central leptin suppresses splenic lymphocyte functions through activation of the corticotropin-releasing hormone-sympathetic nervous system. Brain Res 855(1): 192-197, 2000. PMID: 10650150. DOI: 10.1016/s0006-8993(99)02409-9

13 López M: Hypothalamic AMPK and energy balance. Eur J Clin Invest 48(9): e12996, 2018 PMID: 29999521. DOI: 10.1111/ eci.12996

14 Tanida M and Yamamoto N: Central AMP-activated protein kinase affects sympathetic nerve activity in rats. Neurosci Lett 503(3): 167-170, 2011. PMID: 21893163. DOI: 10.1016/ j.neulet.2011.08.013
15 Paxinos $G$ and Watson $C$ : The Rat Brain in Stereotaxic Coordinates. 6th ed. San Diego, CA, USA, Academic Press, 2007.

16 Ramkumar D and Schulze KS: The pylorus. Neurogastroenterol Motil 17(Suppl 1): 22-30, 2005. PMID: 15836452. DOI: 10.1111/j.1365-2982.2005.00664.x

17 Lerman SH, Mason GR, Bathon EM and Ormsbee HS 3rd: Pyloric motor response to sympathetic nerve stimulation in dogs. Surgery 89(4): 460-465, 1981. PMID: 7209794.

18 Irwin $\mathrm{M}$, Hauger $\mathrm{R}$ and Brown M: Central corticotropinreleasing hormone activates the sympathetic nervous system and reduces immune function: increased responsivity of the aged rat. Endocrinology 131(3): 1047-1053, 1992. PMID: 1505449. DOI: 10.1210/endo.131.3.1505449

19 Tian Z, Sun R, Wei H and Gao B: Impaired natural killer (NK) cell activity in leptin receptor deficient mice: leptin as a critical regulator in NK cell development and activation. Biochem Biophys Res Commun 298(3): 297-302, 2002. PMID: 12413939. DOI: $10.1016 / \mathrm{s} 0006-291 \mathrm{x}(02) 02462-2$

20 Tanida M, Zhang T, Sun L, Song J, Yang W, Kuda Y, Kurata Y and Shibamoto T: Anaphylactic hypotension causes renal and adrenal sympathoexcitaion and induces c-fos in the hypothalamus and medulla oblongata. Exp Physiol 103(6): 790806, 2018. PMID: 29524326. DOI: 10.1113/EP086809

Received August 30, 2019 Revised September 20, 2019 Accepted September 23, 2019 